\title{
Robot-Aided Rehabilitation Methodology for Enhancing Movement Smoothness by Using a Human Hand Trajectory Generation Model With Task-Related Constraints
}

\author{
Yoshiyuki Tanaka \\ Nagasaki University
}

\begin{abstract}
Natural motion produced by the biological motor control system presents movement smoothness, but neurological disorders or injuries severely deteriorates motor functions. This paper proposes a robot-aided training methodology focusing on smooth transient trajectory generation by the arm while performing a complex task (i.e., virtual curling). The aim of the proposed approach is that a trainee should be taught a reference velocity profile with high movement smoothness in the complex task via the interaction with a robotic device while improving coordination ability for natural arm movements. In the virtual curling training, a trainee manipulates the handle of an impedance-controlled robot to move a virtual stone to the center of a circular target on ice while predicting transient behaviors of the released stone. First, a reference hand motion is clarified through a set of preliminary experiments for different task conditions carried out with four welltrained subjects, and the characteristics of skilled hand velocity profiles are coded with a set of quantitative factors as task-related constraints. The skilled hand motions according to task conditions are successfully simulated in the framework of a minimum-jerk model with the taskrelated constraints. Next, the training program for enhancing movement smoothness is developed using the computational model, which has four training modes of operation: 1) diagnosis, 2) teaching with active-assistance by the robot, 3) training with passive-assistance, and 4) training with no assistance. Finally, training experiments with ten novice healthy volunteers demonstrate that the proposed approach can be utilized in the recovery of motor functions necessary for desired velocity profiles with high motion smoothness.
\end{abstract}

Keywords: robot-aided rehabilitation, movement smoothness, trajectory generation

\section{Introduction}

Rapidly aging societies (e.g., in Japan) have witnessed an increase in the number of patients with motor disorders, such as stroke patients who cannot perform natural movements and require motor training for maximum recovery from damage to their neuromuscular system (Ministry of Health, Labour and Welfare, 2007; WHO, 2008). In general, the therapist moves the limb of a patient with motor disorder repeatedly to teach an appropriate smooth movement of the limb in reconstructing patient's motor function. The work load of this hands-on therapeutic technique is difficult. Additionally, the shortage of therapists has been turning into a serious problem in the

\footnotetext{
Authors retain copyright and grant the Journal of Human-Robot Interaction right of first publication with the work simultaneously licensed under a Creative Commons Attribution License that allows others to share the work with an acknowledgement of the work's authorship and initial publication in this journal.
}

Journal of Human-Robot Interaction, Vol. 4, No. 3, 2015, Pages 101-118, DOI 10.5898/JHRI.4.3.Tanaka 
Tanaka, Robot-aided rehabilitation methodology for enhancing movement smoothness

rehabilitation field. Therefore, an advanced training system using robotic devices has been desired to improve the motor functions of patients efficiently and also to reduce the burden on therapists. So far, many robot-assisted training systems have been developed, especially for recovery of upper-limb motor functions. Clinical reports have presented the effectiveness of robot-aided rehabilitation with active/passive assistance, and that significant improvements of motor functions (movement accuracy, speed, and smoothness) for chronic and/or sub-acute patients in a Point-toPoint (PTP) reaching task were correlated to clinical scores, such as Fugl-Meyer Upper Extremity (FMA-UE), Motor Power (MP), and Motor Status Score (MSS) (Arya, Pandian, Verma, \& Garg, 2011; Blank, French, Pehlivan, \& O’Malley, 2014; Kahn, Lum, Rymer, \& Reinkensmeyer, 2010; Maciejasz, Eschweiler, Gerlach-Hahn, Jansen-Troy, \& Leonhardt, 2014; Marchal-Crespo \& Reinkensmeyer, 2009; Masiero et al., 2014; Nordin, Xie, \& Wünsche, 2014).

It is suggested that one of the conclusive motor functions in recovering from motor disorders is movement smoothness. In fact, the successful progress of motor rehabilitation presented that patients' movements became smoother with their recovery while decreasing the value of the jerk metric and finally producing almost single-peak velocity profile in reaching movements, while the recovery of motion smoothness significantly correlates to FMA and MP in PTP tasks (Rohrer et al., 2002). On the analogy of this fact, if a robotic rehabilitation system can provide an appropriate reference velocity profile with high smoothness to task conditions as therapists do for a patient, it can be expected to provide active/passive assistance and to teach on the basis of the reference velocity profile according to individual differences in a trainee's motor abilities. To attain that, robot-aided rehabilitation systems would have to simulate human hand trajectories suitable for performing a training task.

On the other hand, it is well known that the arm of a human without any neurological disorders generates a smooth spatiotemporal trajectory with a bell-shaped velocity profile while performing PTP reaching movements (Georgopoulos, 1986; Morasso, 1981) and several trajectory generation models express such natural motor properties (Flash \& Hogan, 1985; Harris \& Wolpert, 1998; Morasso, Sanguineti, \& Tsuji, 1993; Ohta, Svinin, Luo, Hosoe, \& Laboissire, 2004; Tanaka, Tsuji, Sanguineti, \& Morasso, 2005; Tsuji, Tanaka, \& Kaneko, 2002; Tsuji, Tanaka, Morasso, Sanguineti, \& Kaneko, 2002; Uno, Kawato, \& Suzuki, 1989; Wada \& Kawato, 2004). Some robotic rehabilitation systems have utilized transient behaviors generated by a computational model based on the results of neuroscience studies on reaching movements, as a reference motion to provide active/passive motion assistance according to designed tasks (Maciejasz et al., 2014). However, the previous systems were effective only for simple PTP tasks, such as one-shot reaching movements, and the technical challenge still remains on how to assist or guide trainee's motion in a complex task that involves movements connecting to the activities of daily living (ADL) as repetitive practice (i.e., that will be effective to encourage patients' voluntary efforts in their rehabilitation) (Blank et al., 2014; Marchal-Crespo \& Reinkensmeyer, 2009).

In this study, the robot-aided rehabilitation methodology for enhancing hand movement smoothness is presented as per a virtual curling task and as a complex training task performed by the upper limb. The developed robotic system can teach a reference velocity profile according to individual motor characteristics, such as peak velocity and range of hand motion in PTP movements. It can also provide a set of different training modes for active-assistance, assist-asneeded guidance, and no assistance in training. Experiments with healthy volunteers show that trainees can learn and generate a reference velocity profile with improving their movement smoothness by repetition training with robotic-assistance.

\section{Related Work}

A continuous passive motion (CPM) device (Salter, 1984), which passively moves the joints of a patient, is a pioneering mechatronic-technology-based device for realizing joint motion exercise 
Tanaka, Robot-aided rehabilitation methodology for enhancing movement smoothness

for prevention and improvement of joint contraction and muscle atrophy. Advanced CPM devices using an impedance-controlled method (Hogan, 1985) can execute compliant motion exercises by following the recorded motion of a therapist or a healthy person (Lum, Lehman, \& Reinkensmeyer, 1995; Okada et al., 2001; Okajima et al., 2001). However, CPM did not lead to improvements in motor function, indicating that although this type of therapy has shown possible benefits in neural recovery, it is not sufficient to produce measurable functional benefits (Blank et al., 2014).

Many robotic training systems for the upper limb using the virtual reality technique have also been developed in which a trainee can perform a game-like training task with voluntary rehabilitation efforts (Arya et al., 2011; Blank et al., 2014; Huang \& Krakauer, 2009; Kahn et al., 2010; Maciejasz et al., 2014; Marchal-Crespo \& Reinkensmeyer, 2009; Nordin et al., 2014). The mechanical structure of such rehabilitation robots can be roughly classified into the end-effector type (MIT-MANUS, InMotion2, MIME) and the exoskeletons type (EXO-UL7, Armeo, ARMin) (Arya et al., 2011). Some robotic systems with multiple degrees of freedom provide many handmotion training tasks in a 3D task space (Furusho et al., 2005). One such pioneering robot-aided training system is MIT-MANUS: developed for a training system for improving upper limb movements through operation of the end-effector of an impedance-controlled robot in the horizontal plane, in which a patient is asked to trace a target pattern indicated on the computer display (Krebs, Hogan, Aisen, \& Volpe, 1998). Significant difference between the sub-acute and chronic patients in mean and peak speed of the end-effector in rehabilitations was reported, while improvement of their motor functions relative to movement smoothness was significantly correlated to FMA-UE, MP, and MSS (Rohrer et al., 2002).

Some of the recently developed systems have an intelligent function of motionassistance/guidance with the encouragement of patient's efforts, in which the robot can teach a trainee the simulated hand trajectory as a reference motion for a simple task with single motion based on the patient's motor abilities of the limb and/or the level of task-oriented performances (Amirabdollahian et al., 2003; Erol \& Sarkar, 2007; Krebs et al., 2003; Tanaka, Tsuji, \& Kaneko, 1999; Tsuji, Tanaka, Kaneko, \& Miyaguchi, 2000; Wisneski \& Johnson, 2007). The robotic system that does not utilize a reference trajectory but rather uses a force field for teaching PTP hand movements to a trainee has also been proposed (Mussa-Ivaldi \& Patton, 2000).

Clinical experiments, including at long-term follow-ups, demonstrated the effectiveness of such robot-aided systems in motor recovery of the limb (e.g., Dipietro et al., 2012; Lo et al., 2010), and that robot-assisted therapy can be comparable to conventional rehabilitation therapy in some motor functions. However, the efficacy of robot-aided rehabilitation is still pending, since some experimental reports assess that robotic upper-limb training seems to fail to transfer the wellness improvements of motor function required in performing ADL tasks. It is currently supposed that greater effort should be geared toward providing better assessment solutions to ensure the validity of continuous assessment from robot-assisted rehabilitation (Nordin et al., 2014).

\section{Method}

\subsection{Subjects}

Healthy volunteers (14 male university students, aged 21-24 years, right-handed) participated in this study, and they were provided brief explanations about the aim of the research and about the virtual curling task to be performed by manipulating a robotic device. Four of the volunteers were trained to identify a reference hand motion for the target task, and the others carried out motor training tests for learning the desired hand velocity profile with/without robotic motion assistance.

\subsection{Apparatus}

Fig. 1 shows an overview of the experimental system developed with the robotic device for the virtual curling task. The operator is asked to manipulate the handle to throw a virtual stone that is 
represented using a viscoelasticity model toward the center of a house $O_{h} \in \mathfrak{R}^{2}$, while moving his or her hand slowly and smoothly to stop the stone as near as possible to the center of the house. The operator then predicts the stopping position of stone from the ice condition and the transient behavior of stone in the past along with current turns from the visual feedback information on the display.

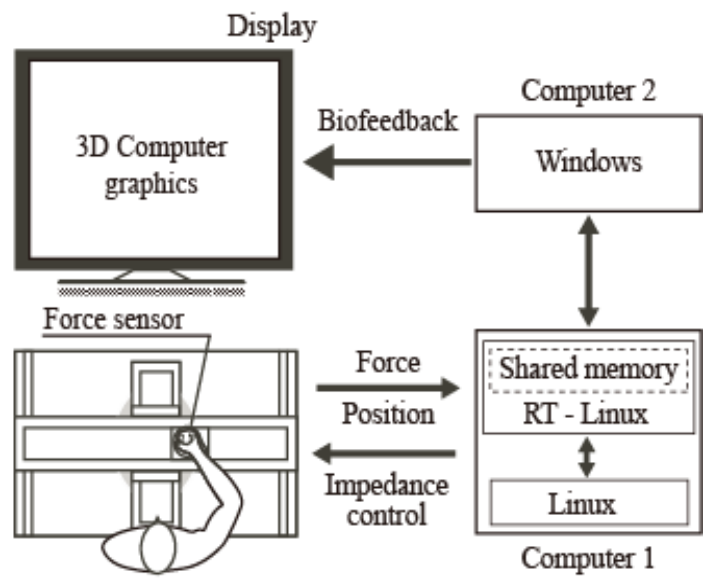

(a)

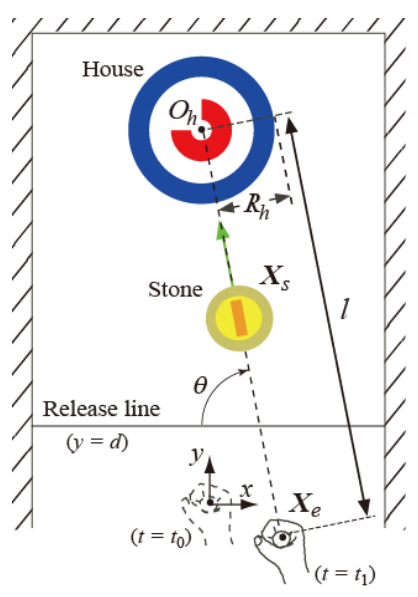

(b)

Figure 1. Experimental apparatus developed for the virtual curling task by the arm. (a) An illustration of the robotic system. The system is composed of an impedance-controlled robotic device to apply virtual force loads on an operator's hand, two computers for robot control and signal processing, and a biofeedback display to present the virtual curling space and training results. (b) A virtual curling model. The virtual curling model is installed on the system, in which the motion of the stone in the virtual space is linked with that of the handle before the stone passes across the release line.

The robotic device has two linear motor tables with one degree of freedom ( $x$ axis: Nihon Thompson Corp., maximum force $\pm 100 \mathrm{~N}$, encoder resolution $2 \mu \mathrm{m} ; y$-axis: Nihon Seikou Co. Ltd., maximum force $\pm 400 \mathrm{~N}$, encoder resolution $1 \mu \mathrm{m}$ ) that are placed orthogonally to allow hand-motion exercises on the horizontal plane. The hand force generated by the operator is measured using a six-axis force/torque sensor (BL Autotec Ltd., resolution: force, $x$ and $y$ axes, $0.05 \mathrm{~N} ; z$ axis, $0.15 \mathrm{~N}$; torque, $0.003 \mathrm{Nm}$ ) attached on the handle of the robot, and the hand position is measured by encoders built in the linear motor tables.

Dynamics of the handle of the impedance-controlled robotic device (Hogan, 1985) is designed according to the handle position, before and after releasing the stone, as follows:

$$
F_{e}= \begin{cases}\left(M_{r}+M_{s}\right) \ddot{X}_{e}+\left(B_{r}+B_{s}\right) \dot{X}_{e} & \left(y \leq d_{y}\right) \\ M_{r} \ddot{X}_{e}+B_{r} \dot{X}_{e} & \left(y>d_{y}\right)\end{cases}
$$


where $F_{e} \in \mathfrak{R}^{2}$ is the force applied by the hand, $X_{e} \in \mathfrak{R}^{2}$ is the hand position, $M_{r}=$ diag. $\left(m_{r}, m_{r}\right) \in$ $\mathfrak{R}^{2 \times 2}$ and $B_{r}=$ diag. $\left(b_{r}, b_{r}\right) \in \mathfrak{R}^{2 \times 2}$ are the handle inertia and viscosity, respectively. $M_{s}=$ diag. $\left(m_{s}\right.$, $\left.m_{s}\right) \in \mathfrak{R}^{2 \times 2}$ is the stone inertia, $B_{s}=\operatorname{diag} .\left(b_{s}, b_{s}\right) \in \mathfrak{R}^{2 \times 2}$ is the viscosity between the stone and the floor. The stone's behavior $X_{s} \in \Re^{2}$ after passing across the release line is given by

$$
M_{s} \ddot{X}_{s}+B_{s} \dot{X}_{s}=0
$$

Accordingly, the stone's stop position can be determined by the stone's release velocity $v_{s}$, as

$$
v_{s}=(l-d) \cdot \frac{b_{s}}{m_{s}}
$$

The trainee is required to adapt his arm motion before releasing the stone and to predict the stone's behavior for the next trial based on the positional error between the stone and the center of the house in the previous trials as well as the dynamic motion of the released stone.

In this study, the distance from the initial position of the stone to the release line is set at $d=$ $0.12 \mathrm{~m}$, considering the reach of the operator's hand. The viscosity between the stone and the floor is set at $b_{s}=1 \mathrm{Ns} / \mathrm{m}$, the handle inertia is set at $m_{r}=5 \mathrm{~kg}$, and the radius of the stone and the center of the house are set at $R_{s}=0.05 \mathrm{~m}$ and $O_{h}=(0,1) \mathrm{m}$, respectively. The house is drawn with concentric circles having radii of $0.05,0.25,0.45$, and $0.65 \mathrm{~m}$, and the task performance $E_{p}$ is scored according to the positional error: 30 points for within $0.25 \mathrm{~m}, 20$ points for within $0.45 \mathrm{~m}$, and 10 points for within $0.65 \mathrm{~m}$.

\subsection{Robot-Aided Training Modes for Enhancing Movement Smoothness}

The developed system facilitates training with four different modes as shown in Fig. 2, as follows:

Diagnosis mode is for self-examining the operator's motor abilities (maximum hand force, hand velocity, and the range of hand motion) during free manipulation of the handle. The diagnosis result will be utilized to simulate a reference motion to the operator.

Teaching mode is to teach an operator the required hand motion with high smoothness in which the handle of robotic device moves to follow the reference trajectory designed based on the operator's motor abilities. This mode aims to challenge motor image training, as the case of active-assistance (Marchal-Crespo \& Reinkensmeyer, 2009).

Passive-assistance mode is to enable an operator to throw the stone straight toward the center of the house. Virtual walls are created with a viscoelastic model in order to control the hand motion perpendicular to the direction toward the center of the house. The operator moves the handle, with no robotic hand-motion assistance, toward the center of the house. This mode aims to inform movement errors from an ideal straight path by providing reaction forces from the virtual walls in movement, as the case of assistance-as-needed (MarchalCrespo \& Reinkensmeyer, 2009).

Training \& evaluation mode is to train a participant with no robotic assistance and to evaluate this trainee's performance of the virtual curling task. 
Tanaka, Robot-aided rehabilitation methodology for enhancing movement smoothness

The adequate combination of these four modes enables progressive learning of the given task by improving hand motor functions for movement smoothness.

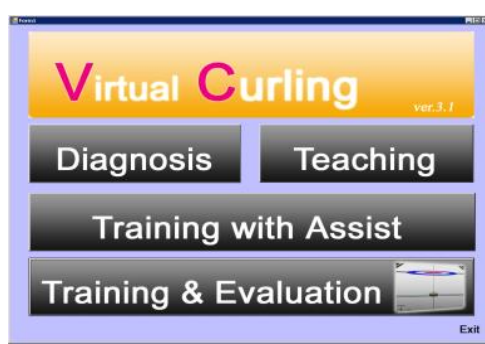

(a)

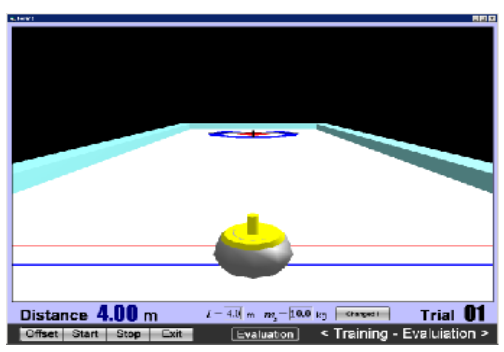

(c)

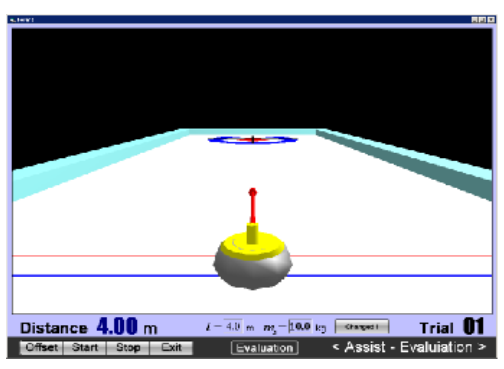

(e)

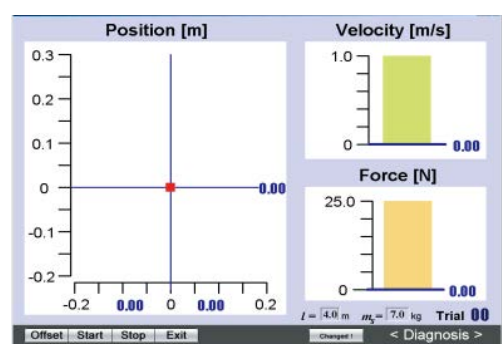

(b)

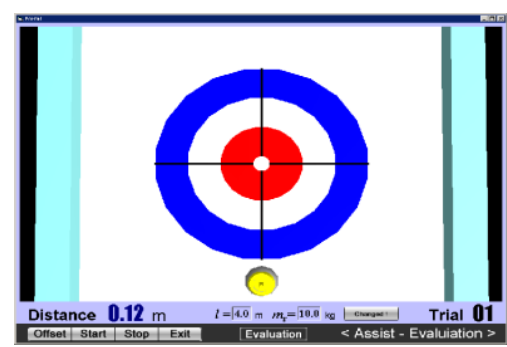

(d)

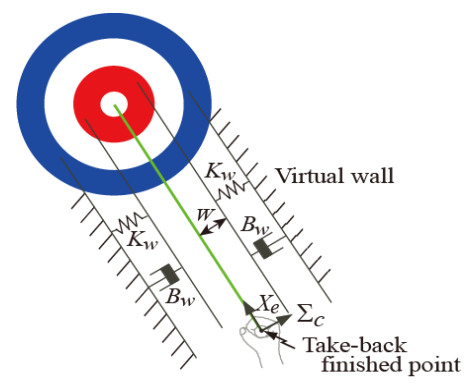

(f)

Figure 2. Computer graphic user interface of the training program for the virtual curling task. (a) Training menu panel, (b) Feedback information in Diagnosis mode. The maximum hand velocity and force during hand motion are presented. (c) Virtual curling space in the Teaching mode and Training \& Evaluation mode. The blue line represents the stone position; the red line represents the release line. (d) The picture when the stone is nearing the house. (e) Virtual curling space in Passive-Assistance mode. The red arrow appears to indicate the motion direction of stone. (f) Illustration of a virtual-wall in Passive-Assistance mode.

\subsection{Minimum-Jerk Trajectory Generation With Task-Related Constraints}

A reference hand motion in the virtual curling task is computed within the framework of the minimum-jerk trajectory generation model by using the optimal control theory with the cost function $J$ given by 
Tanaka, Robot-aided rehabilitation methodology for enhancing movement smoothness

$$
J=\int_{0}^{t_{f}}\left\{\left(\frac{d^{3} x}{d t^{3}}\right)^{2}+\left(\frac{d^{3} y}{d t^{3}}\right)^{2}\right\} d t
$$

under the following task-related boundary conditions:

$$
\left\{\begin{aligned}
\text { Initail }: y\left(t_{0}\right) & =0, \dot{y}\left(t_{0}\right)=0, \ddot{y}\left(t_{0}\right)=0 \\
\text { Take-back }: \dot{y}\left(t_{1}\right) & =v_{1} \\
\text { Release }: y\left(t_{2}\right) & =d, \dot{y}\left(t_{2}\right)=v_{2} \\
\text { Terminal }: y\left(t_{f}\right) & =y_{f}, \dot{y}\left(t_{f}\right)=0, \ddot{y}\left(t_{f}\right)=0
\end{aligned}\right.
$$

where $t_{0}$ and $t_{f}$ are the initial time and the terminal time of hand motion, respectively; $v_{1}$ and $v_{s}$ are the maximum hand velocity during the take-back motion (i.e., the forward swinging motion before releasing the stone and the hand velocity at the release line, respectively); and $y_{f}$ is the terminal hand position along the $y$-axis. The values of constraint parameters depend on the task condition and are determined on the basis of hand-motion trajectories by the well-trained skilled subjects.

\subsection{Procedure}

3.4.1 Reference Motion Detection The four skilled subjects were seated and threw the virtual stone to the center of the house by manipulating the handle of the robotic device with their dominant (right) hand, as shown in Fig. 3. The initial direction between the stone and the house

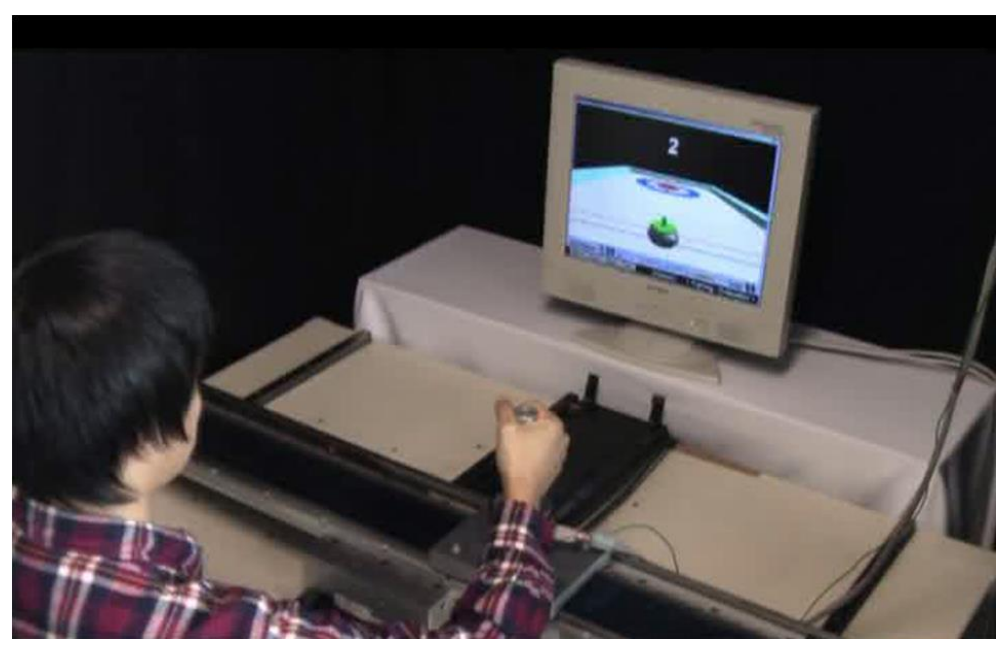

Figure 3. The scene in performing the virtual curling task. The operator starts the task when the number on the display reads zero. The stone position synchronizes with the handle position until the stone is released. 
was set at $\theta=90 \mathrm{deg}$. for this paper (See Fig. 1(b)). The stone inertia $m_{s}$ and the distance $l$, which are the parameters that affect the desired velocity given by Eq. (3), were varied as $m_{s}=7,10$, and $13 \mathrm{~kg}$, and $l=2,3$, and $4 \mathrm{~m}$, respectively. Accordingly, nine conditions were designed for reference motion detection.

Fifteen trials were carried out for each of the nine conditions to detect the reference hand motion, after the subject exhibited the ability to continuously stop the stone within $0.25 \mathrm{~m}$ from the $O_{h}$ several times (at least 3) in the preliminary training.

3.4.2 Motor Training Using Reference Trajectory Ten novice subjects were divided into two groups (Group I: Subs. A-E, and Group II: Subs. F-J). The subjects in Group I performed as per the designed paradigm with the following four steps: (1) four sets of five trials of the virtual curling task in the Training mode, (2) four sets of five trials in the Teaching mode, (3) four sets of five trials in the Passive-Assistance mode, and (4) four sets of five trials in the Training mode. On the other hand, subjects in Group II carried out sixteen sets of five trials in the Training mode. The condition for minimum scoring for skilled subjects was set as $m_{s}=7 \mathrm{~kg}$ and $l=4 \mathrm{~m}$ in the training test. Note that the novice subjects were asked to carry out the curling task with their non-dominant (left) hand.

\subsection{Measures}

3.5.1 Hand motion The hand (handle) position $X_{e}$ and the hand force exerted on the handle $F_{e}$ were recorded with the encoder board and the digital input/output board built into a personal computer (sampling frequency: $1 \mathrm{kHz}$ ), respectively. The hand force measured was filtered out with the low-pass digital filter (cut-off frequency: $25 \mathrm{~Hz}$ ). The terminal hand-motion time $t_{f}$ was calculated as the time when the hand velocity became $0.001 \mathrm{~m} / \mathrm{s}$. The average spatiotemporal trajectory from five trials with higher scores was analyzed and adopted as the skilled hand motion for the designed virtual curling task.

3.5.2 Evaluation Indices Training effectiveness was quantitatively evaluated on the basis of the following two indices:

$$
I_{1}=\frac{p}{30}, \quad I_{2}=\frac{\left(e_{\max }-e\right)}{\left(e_{\max }+e\right)} \cdot r
$$

Index $I_{1}$ is related to the scored point $p$, and index $I_{2}$ is related to the similarity between the measured hand velocity and reference velocity. The parameters $e$ and $r$ denote the squared value of the difference between the measured and reference velocities in motion and the correlation coefficient, respectively. $e_{\max }$ is the value of $e$ in the case when the robot handle is not manipulated. Larger values of the indices indicate better training effectiveness. 
Tanaka, Robot-aided rehabilitation methodology for enhancing movement smoothness

\section{Results}

\subsection{Skilled Hand Trajectory}

Fig. 4 shows typical examples of hand spatial trajectories generated in the preliminary training phase. The hand trajectories sway from side to side in the initial term, but the trajectories converge into a straight line along the $y$-axis as the trial number increases. These were observed for all four subjects.

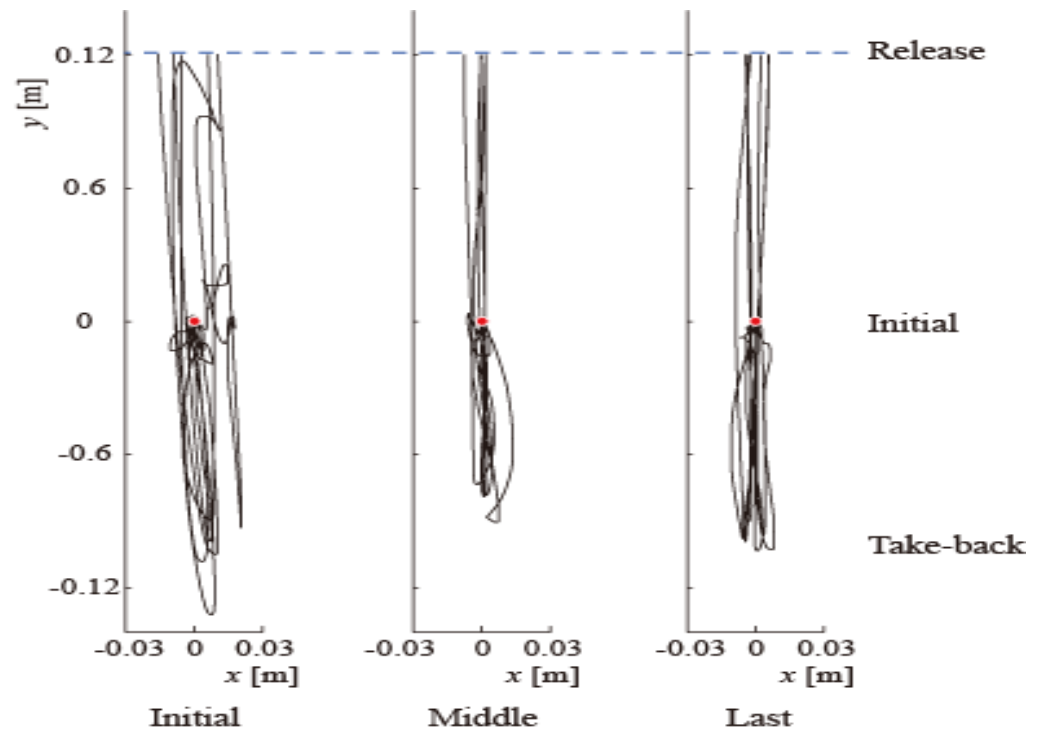

Figure 4. Typical examples of hand trajectories generated just before releasing the stone in the initial term (trials 1-5), the middle term (trials 13-17), and the last term (trials 25-30) of the preliminary training phase by the novice subject. The hand motion became more stable with repetition.

Fig. 5 shows the measured data by the skilled subject for the specified task conditions. The left figures are the results for the distance $l=2,3$, and $4 \mathrm{~m}$ with the stone inertia $m_{s}=10 \mathrm{~kg}$, while the right figures are for the inertia $m_{s}=7,10$, and $13 \mathrm{~kg}$ with the distance $l=3 \mathrm{~m}$. The panels (a) and (b) respectively represent the mean of hand position and velocity profiles in the $y$-axis direction at five trials depending on the task conditions, where the horizontal axis is normalized by the motion time $t_{f}$, and the marker $\nabla$ represent the average of the release time $t_{2}$. The panel (c) represents the summary of transient motion characteristics in the task. The solid line represents the linear approximation of the maximum hand velocity during the take-back motion, and the broken line indicates the computed release velocity expressed by Eq. (3). Both the release velocity and the maximum velocity during take-back motion increase linearly with travel length $l$ but decrease with increasing stone inertia $m_{s}$. It can be seen that the skilled subject released the stone at the computed velocity $\left(v_{s}\right)$ according to the change in $m_{s}$ and $l$. 
Tanaka, Robot-aided rehabilitation methodology for enhancing movement smoothness
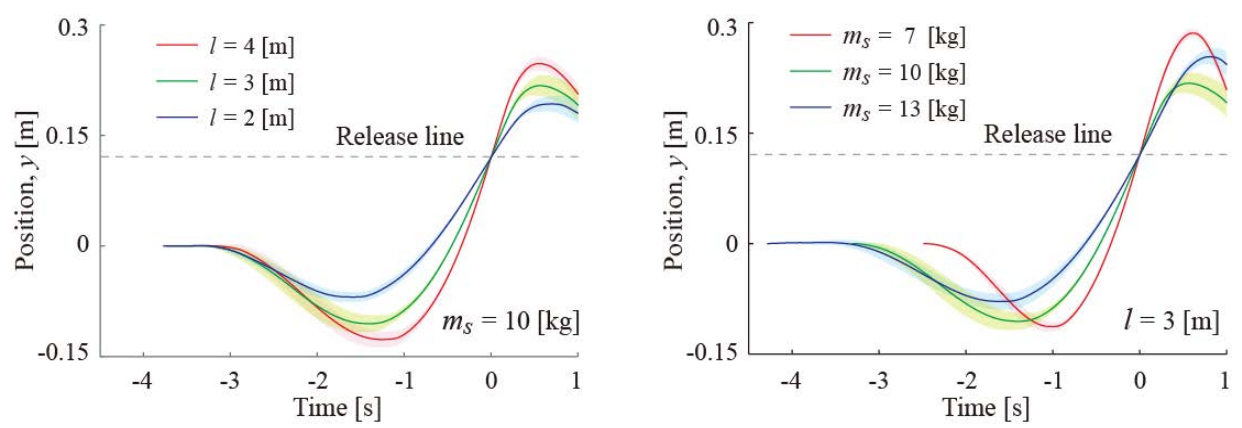

(a)
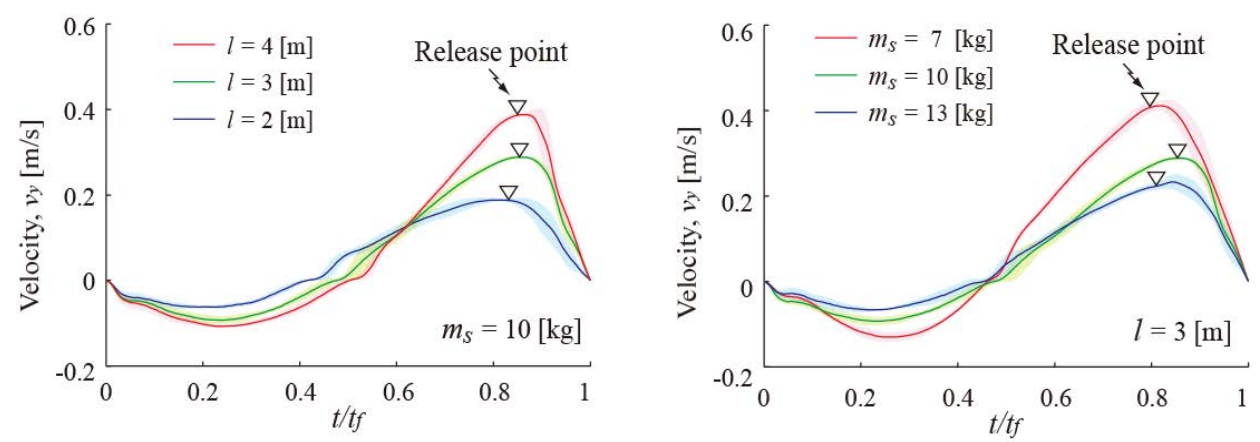

(b)
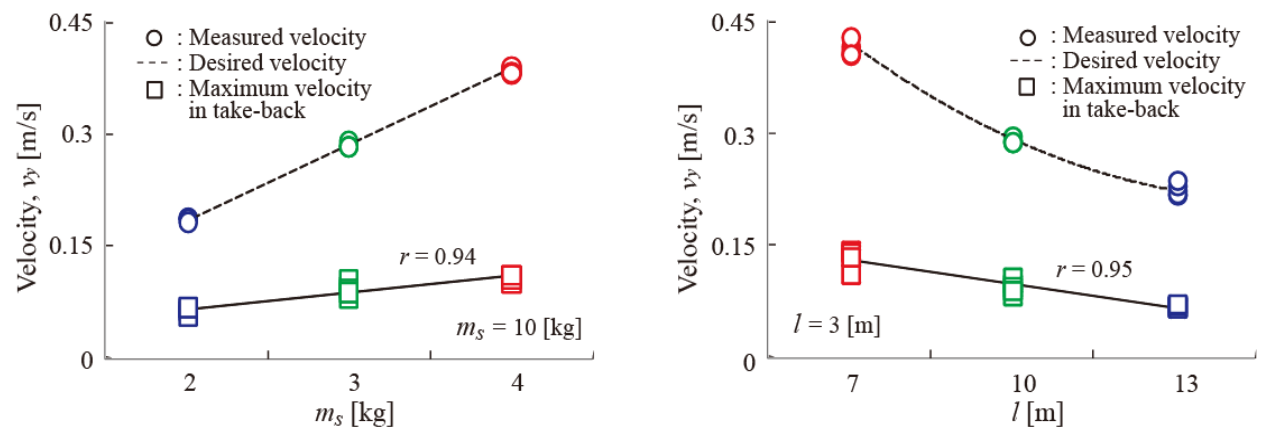

(c)

Figure 5. Changes of hand motion in the $y$-axis direction by skilled subject I for different $l$ and $m_{s}$. The solid line represents the mean of measured data at five trials with higher scores, and the shaded line shows standard deviations. (a) Hand positional profiles. (b) Hand velocity profiles. (c) A set of the release velocity and the maximum hand velocity in the phase of forward swinging motion.

Fig. 6 shows the database of hand-motion characteristics depending on parameters $l$ and $m_{s}$ detected from the measured hand motion for four skilled subjects, in which marker $\odot$ represents the task condition, and data between the white circles was calculated by cubic interpolation. The 

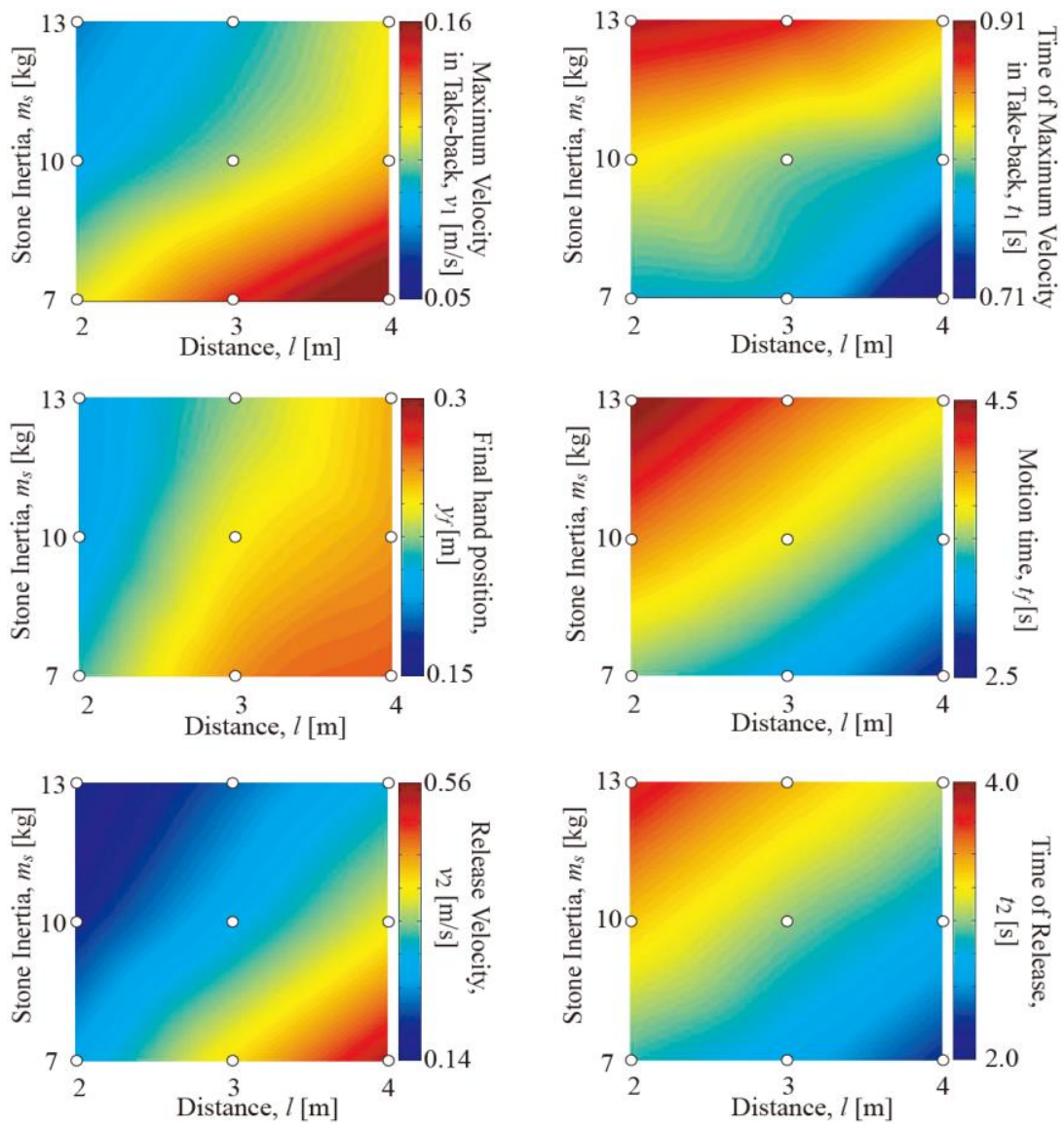

Figure 6. Characteristics of hand movements in the curling task according to different $l$ and $m_{s}$. Each of color maps was made with the mean data of hand trajectories for the four skilled-subjects (white points). These characteristics are utilized as the task-related constraints in generating a hand trajectory for the curling task in the framework of the minimum-jerk model.

maps explain how the well-trained skilled subjects would regulate their hand-motion characteristics to generate a suitable hand spatiotemporal trajectory according to conditions for the virtual curling task.

\subsection{Simulated Hand Velocity Profile With Task-Related Constraints}

Fig. 7 presents a set of simulated hand velocity profiles by using the minimum-jerk model with the task-related constraints given by Eq. (4), in which the values of the constraint parameters were defined with the data maps shown in Fig. 6. Panel (a) shows the change in the velocity profiles depending on $l$ with fixed inertia $m_{s}=10 \mathrm{~kg}$, while panel (b) shows the change depending on $m_{s}$ with fixed distance $l=3 \mathrm{~m}$. It can be seen that the peak and profile of simulated hand velocities depend on the task-related constraints. 


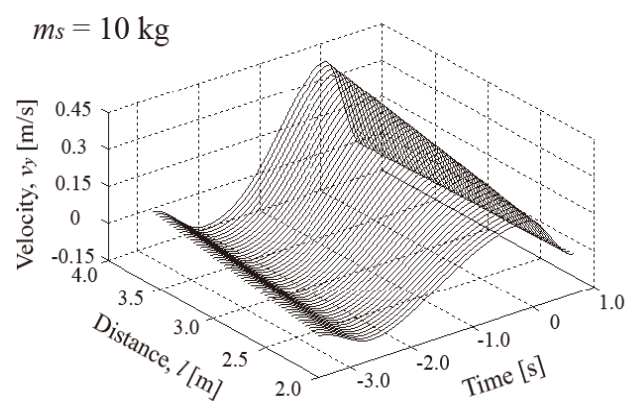

(a)

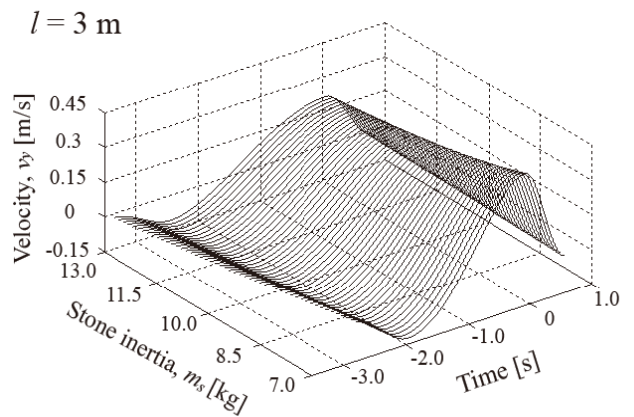

(b)

Figure 7. Simulated hand velocity profiles in the virtual curling task for different $l$ and $m_{s}$ within the designed conditions. (a) Changes in distance $l$ at $m_{s}=10 \mathrm{~kg}$. (b) Changes in stone mass $m_{s}$ at $l=3 \mathrm{~m}$.

\subsection{Training Results}

Fig. 8 shows the trial history of positional error $E_{p}$ in the first and fourth sets obtained by all subjects. $E_{p}$ for the fourth set of Group I is smaller than that of Group II and changes in the same
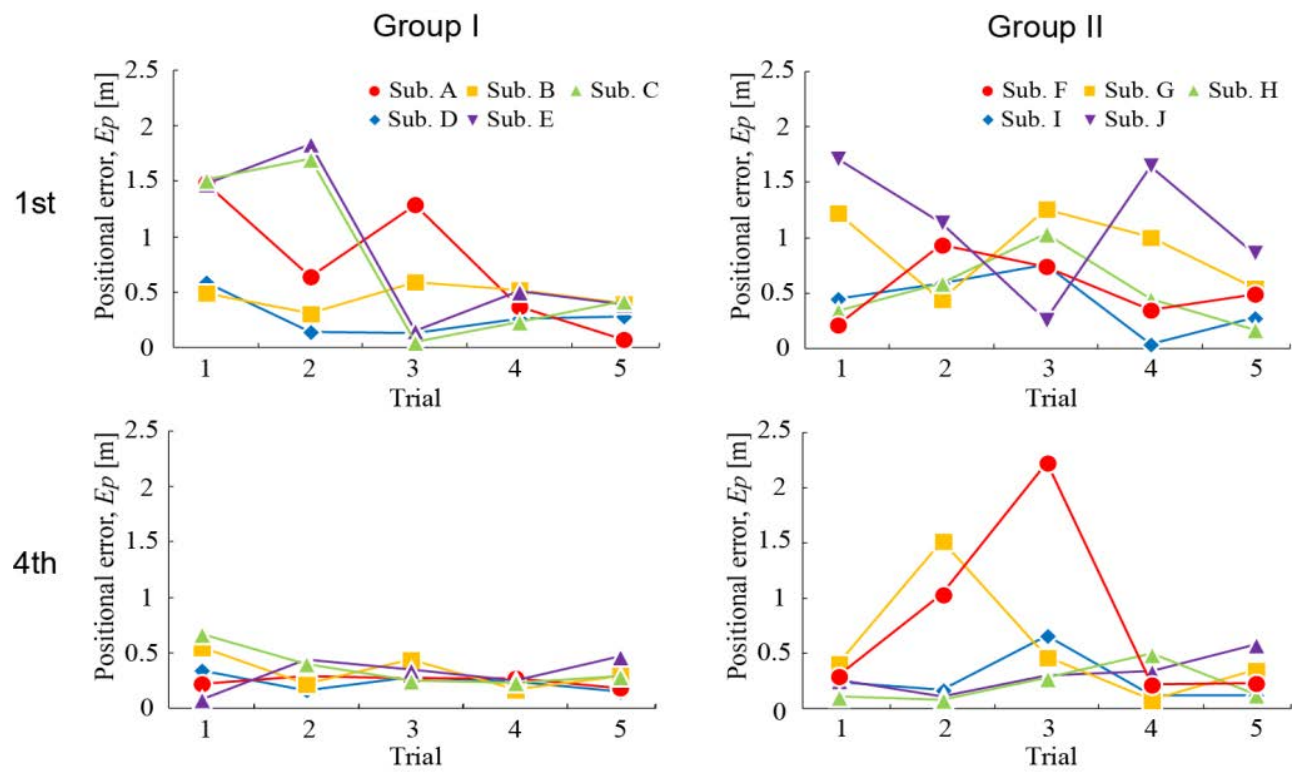

Figure 8. Changes of positional error $E_{p}$ for all subjects in the first and last sets, where errors decrease by repetition, especially in Group I.

way for all subjects. The positional error for Group I reduced as compared to that for Group II in the last set.

Fig. 9 shows the examples of measured-hand velocity profiles for Sub. A (Group I) and Sub. F (Group II) in the first and fourth sets. Hand motions for the two subjects are similar in the initial set, and remarkable differences between them appear after the second set. Sub. A constantly 
generates hand velocity profiles in good agreement with the reference profile after having active and passive assistance provided by the robot, but Sub. F does not.
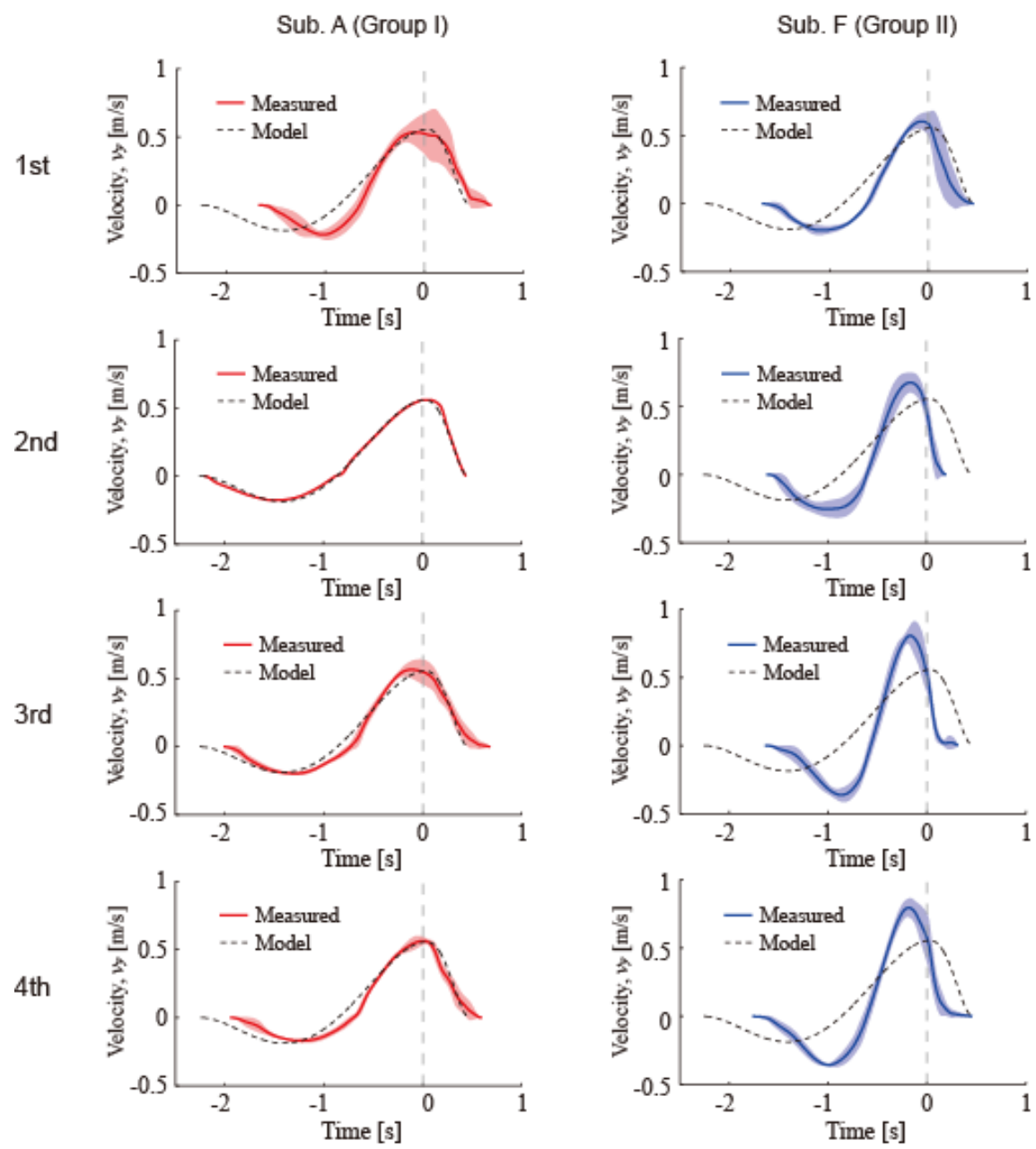

Figure 9. Measured and reference hand velocities for Subs. A (Group I) and F (Group II), where the subjects executed the tests with their non-dominant (left) hand. The solid line represents the mean of measured data at five trials, the broken line represents the reference velocity profile for the task condition of stone mass $m_{s}=7 \mathrm{~kg}$ and distance $l=4 \mathrm{~m}$, and the shaded line shows the standard deviations.

Fig. 10 presents calculation results of the jerk metrics for each of the two groups in the first and last sets and for the four skilled subjects. The values of the jerk metric were reduced (i.e., motion smoothness was improved by the repetition of training for both groups and relatively for Group I. Since the jerk metric for Group I in the last set is close to that of the skilled subjects, the subjects of Group I moved their non-dominant hand as skillfully and smoothly in the last set as the skilled subjects did by their dominant hand. 
Tanaka, Robot-aided rehabilitation methodology for enhancing movement smoothness

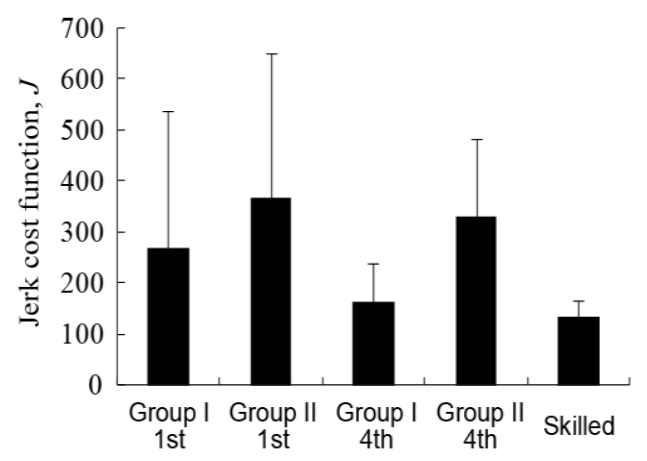

Figure 10. Calculation results of the Jerk cost function from the hand trajectories measured in the first and last sets for each of the two groups and those from the better trajectories for the skilled subjects measured in reference motion detection. Mean and standard values are presented.

Fig. 11 shows evaluation results based on the two indices for performance of the virtual curling task. Performance was improved through training in both groups, and significant differences were noted in the $I_{2}$ value between the groups. This means that not only task scores but also motor abilities for reproducing the reference velocity profile with high smoothness improved for the subjects in Group I who underwent training with robotic assistance based on a biological trajectory generation model.

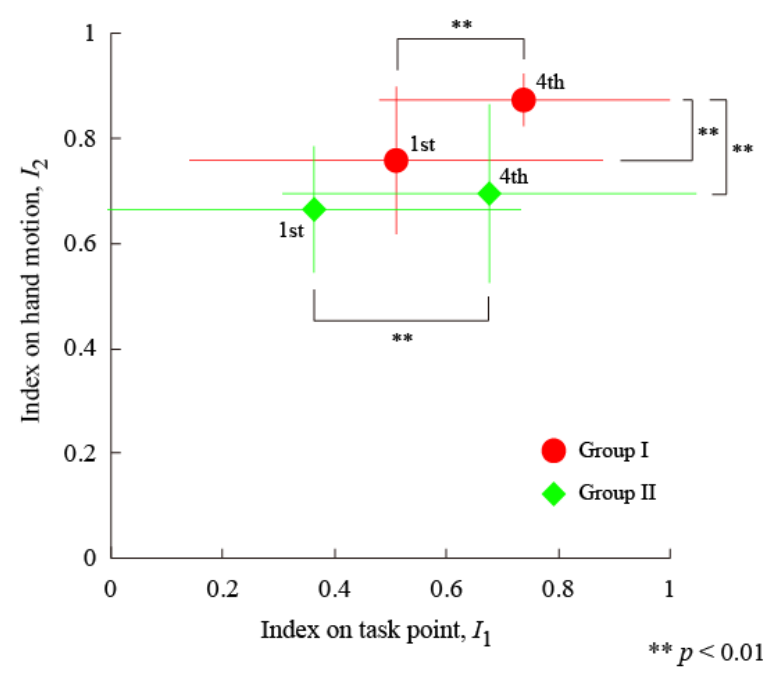

Figure 11. Evaluation results of task performance for each of the two groups at the first and last sets. Mean of the evaluation values for the five subjects was calculated. The horizontal axis represents the point obtained, while the vertical axis represents agreement between the measured hand velocity profiles and the reference profile. 
Tanaka, Robot-aided rehabilitation methodology for enhancing movement smoothness

\section{Discussion}

The present paper proposed training methodology for enhancing movement smoothness for the robot-aided neurorehabilitation system using the impedance controller. The virtual curling task was designed as an example of complex training tasks by the upper limb compared to simple point-to-point (PTP) reaching movements, in which a trainee must operate the robot's handle smoothly while simulating the behavior of a virtual stone after release, so that the stone would stop at the center of the house.

First, we investigated a skillful hand motion in the virtual curling task to use a reference movement in robot-aided rehabilitation and found that well-trained human operators generate a unique hand trajectory with a smooth velocity profile depending on task conditions. Such a skilled hand trajectory was characterized by the task-related spatiotemporal constraints (i.e., maximum hand velocity during take-back motion $v\left[t_{1}\right]$, hand velocity at the release line $v\left[t_{2}\right]$, and the final hand position $\left.y\left[t_{f}\right]\right)$. The reference velocity profile was successfully simulated in the framework of a minimum-jerk model by using the dataset of motor characteristics.

Next, a set of training experiments was carried out to demonstrate the efficacy of the developed velocity-based training program with teaching and passive-assistance (assist-as-needed) modes in improving trainee's temporal movements and task performance in virtual curling. The developed training program significantly helped novice operators to display better task performance with skillful hand transient motion, as observed in the case of the well-trained subjects. It suggested that trainees received an ideal motor image of their arm movements in the teaching mode, while their motion errors from the reference are routed haptically in the passiveassistance mode. This implies that information on transient motion is necessary to impart taskrelated motion skill to a novice operator and to improve motor control ability that relies on the neural mechanism for motor image as well as motor learning centered on the brain. Some recent neuroscience researchers report that such cognitive processing relative to motor actions leads to activate both the cerebellar and cerebral cortexes (Arya et al., 2011; Masiero et al., 2014). Accordingly, the possibility can be also suggested that the proposed velocity-based training program may contribute to train some motor functions owing to the cerebellum.

Fig. 12 summarizes the trajectory generation mechanism for the virtual curling task, including that for the process within the framework of a minimum-jerk model with the task-related constraints, on the basis of biological motor control system factors in an object manipulation task (Davidson \& Wolpert, 2005; Zago, McIntyre, Senot, \& Lacquaniti, 2008).

When a human begins to perform voluntary movements, the cerebral cortex perceives the external environment by reducing redundancy and integrating a variety of information from the sensory organs. In the virtual curling task, it can be said that the cerebral cortex perceives visual information about the virtual space, arm motion, and reaction force from the robot, and collates information on how the virtual stone will move according to the control input for operating the robot handle before and after the stone is released. When the trainees do not successfully acquire an ideal motion to realize best performance (i.e., for the stone to stop at the center of the house), they have to learn the ideal motion by trial and error. Various proposals have been made regarding such a trial and error learning mechanism. Schultz, Dayan, and Montague (1997) suggested that it represents the reward prediction error made by striatal neurons in the basal ganglia. Doya (2000) proposed that the reward prediction model used to select the motion is represented in the striatum and suggested the possibility that reward prediction affects motor learning in the cerebellum. According to these hypotheses, if the distance (error) between the center of the house and the released stone's stop position is assumed to correlate with the reward in the curling task, it can be thought that the trainee plans the ideal motion and controls the body according to the distance obtained in every trial.

In the experiments, the skilled subject's hand motion in the virtual curling task was expressed within the framework of the minimum-jerk model with the task-related constraints. The 
Tanaka, Robot-aided rehabilitation methodology for enhancing movement smoothness

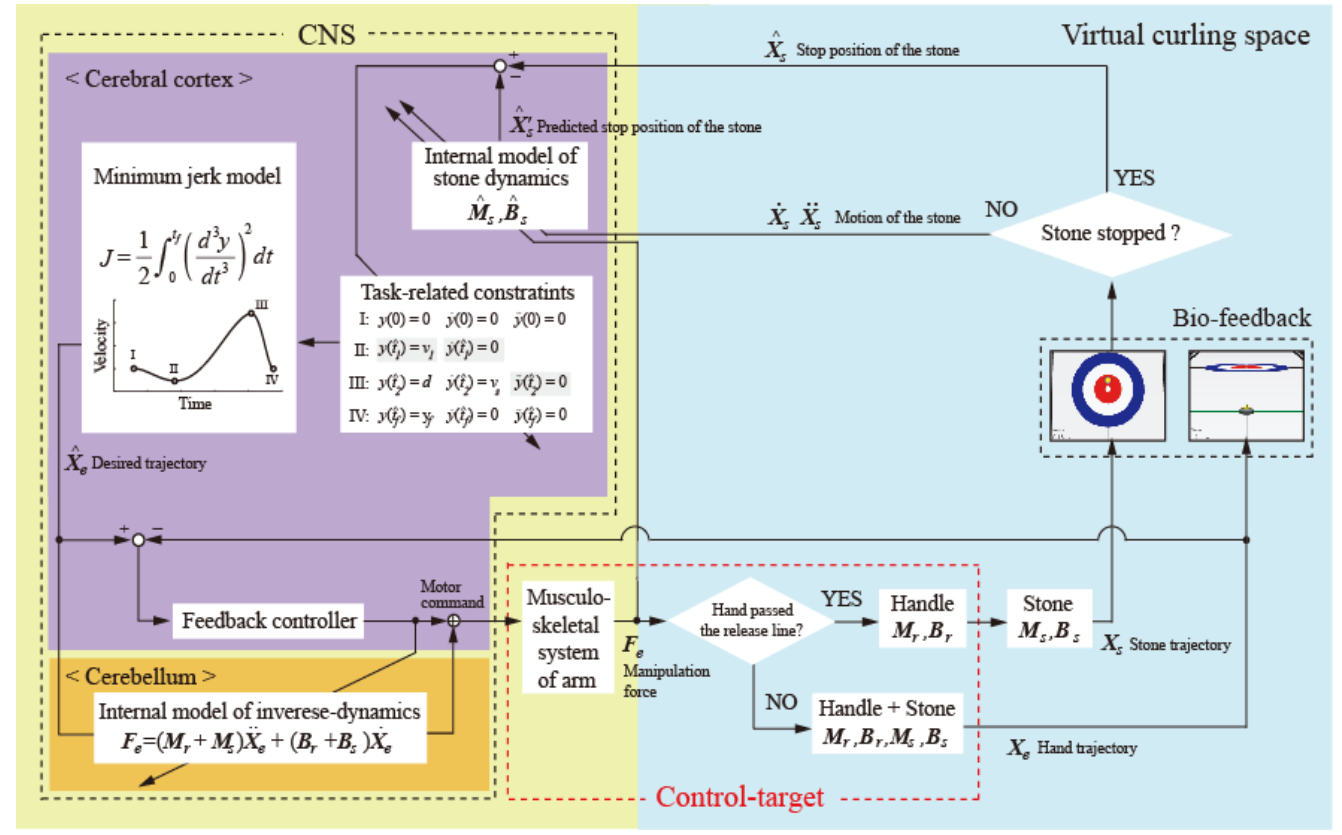

Figure 12. A block diagram for the adaptive motor control system of hand trajectory formation for the virtual curling task.

identification of task-related constraints is related to the reduction in motion redundancy. Therefore, it can be said that the skilled subject uses the cerebral cortex to learn the virtual stone's dynamics by repeating the task according to the information regarding the error between the predicted stop position and the actual stop position, and that they accurately predict the position where the stone will stop. As the subjects experienced the virtual curling task, they generated an almost unique smooth velocity wave in arm motion. These results come from their ability to predict the controlled object's motion, while it is known that an internal representation of the controlled object's properties is necessary to make such a prediction. This internal representation is called the internal model, and various proposals about the internal model in the cerebellum have been made. For example, researchers proposed the feed-forward control model, in which the inverse dynamics of a controlled object is acquired in the cerebellum (Kawato, 1999; Kawato et al., 2003; Wolpert, Miall, \& Kawato, 1998). It is also known that the cerebellum has a large role in realizing optimal motion by using the musculoskeletal system (Topka, Konczak, \& Dichgans, 1998). In the virtual curing task, the trainee has to learn inverse dynamics of the controlled objects, such as the impedance-controlled robot handle and the musculoskeletal system only by repeating the task, and the influence of feedback control in the cerebral cortex becomes smaller as the optimal inverse dynamics model is based in the cerebellum. Therefore, it can be said that human trainees acquired the ability to perform the feed-forward control that can generate an ideal motion command for the musculoskeletal system after successfully undergoing training for realizing smooth motions with the proposed velocity-based methodology in robotic rehabilitation.

Clinical reports present that sub-acute and chronic patients with motor disorders generate a jagged movement in PTP reaching tasks, while their movements become smoother with their recovery with decreasing the value of the jerk metric. Additionally, their recovery of motion smoothness significantly correlates to FMA and MP (Rohrer et al., 2002). Therefore, the proposed 
Tanaka, Robot-aided rehabilitation methodology for enhancing movement smoothness

velocity-based methodology will be effective to improve such clinical scores, especially relative to the coordination and speed of arm movements; however, clinical efficacy must be investigated via clinical experiments. Future research will be conducted to determine the target type and level of motor disorders for the proposed velocity-based methodology in the curling task and another complex task. Continued research may also improve usability and flexibility of the robotic system in consideration of a therapists' background knowledge on the advanced technology, so that therapists can customize training protocol and task difficulty for individuals with motor disorders.

\section{Acknowledgements}

The author greatly thanks Mr. T. Sanemasa for his many contributions in this research work and Prof. T. Tsuji for his comments. This work was partly supported by JSPS KAKENHI (16760203).

\section{References}

Amirabdollahian, F., Loureiro, R., Gradwell, E., Collin, C., Harwin, W., \& Johnson, G. (2003). Multivariate analysis of the Fugl-Meyer outcome measures assessing the effectiveness of GENTLE/S robot-mediated stroke therapy. Journal of NeuroEngineering and Rehabilitation, 4(4). doi:10.1186/1743-0003-4-4

Arya, K. N., Pandian, S., Verma, R., \& Garg, R. K. (2011). Movement therapy induced neural reorganization and motor recovery in stroke: a review. Journal of Bodywork and Movement Therapies, 15, 528-537.

Blank, A. A., French, J. A., Pehlivan, A. U., \& O’Malley, M. K. (2014). Current trends in robotassisted upper-limb stroke rehabilitation: Promoting patient engagement in therapy. Current Physical Medicine and Rehabilitation Reports, 2(3), 184-195.

Davidson, P., \& Wolpert, D. (2005). Widespread access to predictive models in the motor system: A short review. Journal of Neural Engineering, 2, S313-S319.

Dipietro, L., Krebs, H., Volpe, B., Stein, J., Bever, C., Mernoff, S., Fasoli, S. E., \& Hogan, N. (2012). Learning, not adaptation, characterizes stroke motor recovery: Evidence from kinematic changes induced by robot-assisted therapy in trained and untrained task in the same workspace. IEEE Transactions on Neural Systems and Rehabilitation Engineering, $20(1), 48-57$.

Doya, K. (2000). Complementary roles of basal ganglia and cerebellum in learning and motor control. Current Opinion in Neurobiology 10, 732-739.

Erol, D., \& Sarkar, N. (2007). Intelligent control for robotic rehabilitation after stroke. Journal of Intelligent and Robotic Systems, 50(4), 341-360.

Flash, T., \& Hogan, N. (1985). The coordination of arm movements: An experimentally confirmed mathematical model. The Journal of Neuroscience, 5(7), 1688-1703.

Furusho, J., Koyanagi, K., Nakanishi, K., Ryu, U., Takenaka, S., Inoue, A., Domen, K., \& Miyakoshi, K. (2005). Development of a 3-D rehabilitation system for upper limbs using ER actuators in NEDO project. International Journal of Modern Physics B, 19(7-9), 1591-1597.

Georgopoulos, A. P. (1986). On reaching. Annual Review of Neuroscience, 9, 147-170.

Harris, C., \& Wolpert, D. (1998). Signal-dependent noise determines motor planning. Nature, 394, 780-784.

Hogan, N. (1985). Impedance control: An approach to manipulation: Parts I, II, III. ASME Journal of Dynamic Systems, Measurement, and Control, 107(1), 1-24. 
Tanaka, Robot-aided rehabilitation methodology for enhancing movement smoothness

Huang, V., \& Krakauer, W. (2009). Robotic neurorehabilitation: A computational motor learning perspective. Journal of NeuroEngineering and Rehabilitation, 6(5). doi:10.1186/17430003-6-5

Kahn, L. E., Lum, P. S., Rymer, W. Z., \& Reinkensmeyer, D. J. (2010). Robot-assisted movement training for the stroke-impaired arm: Does it matter what the robot does? Journal of Rehabilitation Research and Development, 43(5), 619-630.

Kawato, M. (1999). Internal models for motor control and trajectory planning. Current Opinion in Neurobiology, 9, 718-727.

Kawato, M., Kuroda, T., Imamizu, H., Nakano, E., Miyauchi, S., \& Yoshioka, T. (2003). Internal forward models in the cerebellum: fMRI study on grip force and load force coupling. Progress in Brain Research, 142, 171-188.

Krebs, H. I., Hogan, N., Aisen, M. L., \& Volpe, B. T. (1998). Robot-aided neurorehabilitation. IEEE Transactions on Rehabilitation Engineering, 6(1), 75-87.

Krebs, H. I., Palazzolo, J. J., Dipietro, L., Ferraro, M., Krol, J., Rannekleiv, K., Volpe, B. T., Hogan, N. (2003). Rehabilitation robotics: Performance based progressive robot-assisted therapy. Autonomous Robots, 15(7).

Lo, A., Guarino, P., Richards, L., Haselkorn, J., Wittenberg, G., Federman, D., et al. (2010). Robot-assisted therapy for long-term upper-limb impairment after stroke. The New England Journal of Medicine, 362(19), 1772-1783.

Lum, P. S., Lehman, S. L., \& Reinkensmeyer, D. J. (1995). The bimanual lifting rehabilitator: An adaptive machine for therapy of stroke patients. IEEE Transactions on Rehabilitation Engineering, 3(2), 166-173.

Maciejasz, P., Eschweiler, J., Gerlach-Hahn, K., Jansen-Troy, A., \& Leonhardt, S. (2014). A survey on robotic devices for upper limb rehabilitation. Journal of NeuroEngineering and Rehabilitation, 11(3). doi:10.1186/1743-0003-11-3

Marchal-Crespo, L., \& Reinkensmeyer, D. J. (2009). Review of control strategies for robotic movement training after neurologic injury. Journal of NeuroEngineering and Rehabilitation, 6(20). doi:10.1186/1743-0003-6-20

Masiero, S., Poli, P., Rosati, G., Zanotto, D., Iosa, M., Paolucci, S., \& Morone, G. (2014). The value of robotic systems in stroke rehabilitation. Expert Review of Medical Devices, 11(2), 187-198.

Ministry of Health, Labour and Welfare (2007). In Health Statistics in Japan 2007. Retrieved from http://www.mhlw.go.jp/english/database/db-hss/dl/hs2007a.pdf

Morasso, P. G., Sanguineti, V., \& Tsuji, T. (1993). A dynamical model for the generator of curved trajectories. In Proceedings of the International Conference on Artificial Neural Networks (Vol. 394, p. 115-118).

Morasso, P. G. (1981). Spatial control of arm movements. Experimental Brain Research, 42, 223-227.

Mussa-Ivaldi, F. A., \& Patton, J. L. (2000). Robots can teach people how to move their arm. In Proceedings of the 2000 IEEE International Conference on Robotics and Automation ( $\mathrm{p}$. 300-305).

Nordin, N., Xie, S. Q., \& Wünsche, B. (2014). Assessment of movement quality in robot-assisted upper limb rehabilitation after stroke: A review. Journal of NeuroEngineering and Rehabilitation, 11(137). doi:10.1186/1743-0003-11-137

Ohta, K., Svinin, M., Luo, Z., Hosoe, S., \& Laboissire, R. (2004). Optimal trajectory formation of constrained human arm reaching movements. Biological Cybernetics, 91(1), 773-779.

Okada, S., Sakaki, T., Hirata, R., Okajima, Y., Uchida, S., \& Tomita, Y. (2001). TEM: a therapeutic exercise machine for the lower extremities of spastic patients. Advanced Robotics, 14(7), 597-606. 
Tanaka, Robot-aided rehabilitation methodology for enhancing movement smoothness

Okajima, Y., Tanaka, N., Hasegawa, M., Uchida, N., Kimura, A., Tomita, Y., . . . Sakaki, T. (2001). Therapeutic exercise machine: Soft motion by the impedance control mechanism. Sogo Rehabilitation (in Japanese), 26(4), 363-369.

Rohrer, B., Fasoli, S., Krebs, H. I., Hughes, R., Volpe, B., Frontera, W. R., Stein, J., \& Hogan, N. (2002). Movement smoothness changes during stroke recovery. The Journal of Neuroscience, 22(18), 8297-8304.

Salter, R. (1984). Clinical application of basic research on continuous passive motion for disorders and injury of synovial joints: A preliminary report of a feasibility study. Journal of Orthopadic Research, 3, 325-342.

Schultz, W., Dayan, P., \& Montague, P. R. (1997). A neural substrate of prediction and reward. Science, 275(5306), 1593-1599.

Tanaka, Y., Tsuji, T., \& Kaneko, M. (1999). A bio-mimetic rehabilitation aid for human movements using mechatronic technology. In Proceedings of the 1999 International Conference on Mechatronic Technology (p. 245-250).

Tanaka, Y., Tsuji, T., Sanguineti, V., \& Morasso, P. G. (2005). Bio-mimetic trajectory generation using a neural time base generator. Journal of Robotic Systems, 22(11), 625-637.

Topka, H., Konczak, J., \& Dichgans, J. (1998). Coordination of multi-joint arm movements in cerebellar ataxia: Analysis of hand and angular kinematics. Experimental Brain Research, 119(4), 483-492.

Tsuji, T., Tanaka, Y., \& Kaneko, M. (2002). Biomimetic trajectory generation based on human movements with a nonholonomic constraint. IEEE Transactions on Systems, Man, and Cybernetics-Part A: Systems and Humans, 32(6), 773-779.

Tsuji, T., Tanaka, Y., Kaneko, M., \& Miyaguchi, H. (2000). A bio-mimetic rehabilitation aid for reaching movements using time base generator. International Journal of Machine Intelligence and Robotic Control, 2(4), 141-149.

Tsuji, T., Tanaka, Y., Morasso, P. G., Sanguineti, V., \& Kaneko, M. (2002). Bio-mimetic trajectory generation of robots via artificial potential field with time base generator. IEEE Transactions on Systems, Man, and Cybernetics-Part C: Applications and Reviews, 32(4), 426-439.

Uno, Y., Kawato, M., \& Suzuki, R. (1989). Formation and control of optimal trajectory in human multijoint arm movement. Biological Cybernetics, 61(2), 89-101.

Wada, Y., \& Kawato, M. (2004). A via-point time optimization algorithm for complex sequential trajectory formation. Neural Networks, 17(1), 353-364.

WHO (2008). In The global burden of disease: 2004 update. Retrieved from http://www.who.int/healthinfo/global_burden_disease/2004_report_update/en/

Wisneski, K., \& Johnson, M. (2007). Quantifying kinematics of purposeful movements to real, imagined, or absent functional objects: Implications for modelling trajectories for robotassisted ADL tasks. Journal of NeuroEngineering and Rehabilitation, 4(7). doi:10.1186/1743-0003-4-7

Wolpert, D., Miall, R., \& Kawato, M. (1998). Internal models in the cerebellum. Trends in Cognitive Sciences, 2(9), 338-347.

Zago, M., McIntyre, J., Senot, P., \& Lacquaniti, F. (2008). Internal models and prediction of visual gravitational motion. Vision Research, 14, 1532-1538.

Yoshiyuki Tanaka, Graduate School of Engineering, Nagasaki University, Nagasaki, Japan. Email: ytnk@nagasaki-u.ac.jp 